\title{
Effect of Vanadium Compounds on the Lipid Organization of Liposomes and Cell Membranes
}

\author{
Laura BaKás, ${ }^{*}, 1,2$ Georgina Verza, ${ }^{1}$ And Ana Cortizo ${ }^{2}$ \\ ${ }^{1}$ Instituto de Investigaciones Bioquímicas La Plata (INIBIOLP) \\ Facultad de Ciencias Medicas, 60 y 120, (1900) La Plata, \\ Argentina; and ${ }^{2}$ Departamento de Ciencias Biológicas, \\ Facultad de Ciencias Exactas, Universidad Nacional \\ de La Plata, 47 y 115, (1900) La Plata, Argentina
}

Received December 13, 1999; Revised May 9, 2000; Accepted May 20, 2000

\begin{abstract}
The influence of vanadate on the adsorption properties of Merocyanine 540 (MC540) to UMR cells was studied by means of specrofluorometry. An increment in the fluorescence was observed in the osteoblasts incubated with $0.1 \mathrm{mM}$ vanadate. This effect could be interpreted in terms of vanadate inhibitory effects on aminotraslocase activity. However, vanadate promotes a similar behavior to that found in UMR 106 cells when it was added to lipid vesicles composed of phosphatidylcholine. The effect of vanadium in different oxidation states, such as vanadate $(\mathrm{V})$ and vanadyl(IV) on lipid membrane properties was examined in large unilamellar vesicles by means of spectrofluorometry employing different probes. Merocyanine 540 and 1,6-diphenylhexatriene were used in order to sense the changes at interfacial and hydrophobic core of membranes, respectively. In contrast to vanadate, vanadyl decreased the fluorescence of MC540. Both vanadium compounds slightly perturbed the hydrocarbon core. The results can be interpreted by the specific adsorption of both compounds on the polar head groups of phospholipid and suggest a possible influence of vanadium compounds on the lipid organization of cell membranes.
\end{abstract}

Index Entries: Vanadium compounds; liposomes; lipid membranes; membrane fluidity; Merocyanine 540; 1,6-diphenylhexatriene.

*Author to whom all correspondence and reprint requests should be addressed. 


\section{INTRODUCTION}

Vanadium is a transition element present in trace amounts in the atmosphere and in food. At present, it has been established that vanadium has biological functions in lower forms of life; its essentiality for higher animals and human has been accepted. Vanadium deficiency causes growth arrest and skeletal deformation in animals (1). However, the essentiality of vanadium in humans has been questioned, because of the lack of its defined and proven biological role. Different forms of this element induce metabolic and mitogenic events, promoting proliferative processes, acting upon cellular differentiation and producing morphological changes of culture cells (2). Many studies developed in vitro have shown that vanadium acts as a growth factor mimetic compound on osteoblastlike cells $(3,4)$. On the other hand, the vanadium insulinmimetic properties have been recently shown; they include glucose transport and metabolism, ion and amino acidic transport, lipid and glycogen metabolism, gene transcription, and protein synthesis (5). These effects may be characterized according to their induction period, being the early events (after some seconds or minutes) mediated by the interaction of different vanadium species with the plasma membrane.

On the other hand, it has been previously reported that vanadium compounds increase insulin sensitivity by enhancing insulin-binding capacity in rat adipocytes (6). Furthermore, vanadate potentiates the glucose effects on insulin release, an effect that seems to be the result of changes in the membrane potential. In addition, it was observed in early in vitro studies that vanadate strongly inhibits $\mathrm{Na}^{+}-\mathrm{K}^{+}$-ATPase, $\mathrm{Ca}^{2+}$-ATPase, and aminotraslocase activities. However, direct effects produced by vanadate on the membrane lipid phase have not been fully studied (7).

It has been postulated that the potency of the biological effects of vanadium compounds could depend either on the oxidation state or on the coordination geometry of vanadium. Vanadyl $\left(\mathrm{VO}^{2+}\right)$ and vanadate $\left(\mathrm{H}_{2} \mathrm{VO}_{4}^{-}\right)$are the major species of vanadium(IV) and vanadium(V), respectively, that are present in aqueous solution under physiological conditions. These vanadium species interact with a variety of ligands such as carboxyl, phosphate, and amino groups (8-11). Under physiological conditions, vanadate $(\mathrm{V})$ exists in several forms depending on vanadium concentration, $\mathrm{pH}$, and other environmental factors (presence of reductants, ligands, etc).

In the present study, we investigated the effects of vanadium compounds on osteoblastlike cells as well as their interaction with membrane model systems. The membrane fluidity was estimated on the steady-state fluorescence of diphenylhexatriene incorporated into the hydrophobic bilayer and the lipid organization at the membrane interface by means of the fluorescence intensity of the Merocyanine 540 monomer. 


\section{MATERIALS AND METHODS}

\section{Materials}

1-Palmitoyl-2-oleoyl-phosphatidylcholine (POPC) and phosphatidylserine (PS) were obtained from Avanti Polar Lipids, Inc (Birmingham, AL). Thin-layer chromatography using a chloroform : water : methanol $(65: 25: 25)$ mixture gave a single spot under iodine vapors. Therefore, lipids were used without further purification. Merocyanine 540 (MC540) and 1,6diphenylhexatriene (DPH) were obtained from Molecular Probes, Eugene (OR). All other chemicals were of analytical grade.

Tissue culture materials were provided by Corning Dubelcco's modified Eagle's medium (DMEM), trypsin was supplied by Gibco, and fetal bovine serum (FBS) by Gen (Argentina)

All other reagent were of analytical grade.

\section{Cell Culture}

The rat osteosarcoma cell line UMR 106 in DMEM supplemented with 10\% FBS and antibiotics in a humidified atmosphere of $95 \%$ air/ $5 \% \mathrm{CO}_{2}$ (3) was grown. Cells were grown at near confluence $(70-80 \%)$ and they were subcultured using $0.1 \%$ trypsin- $1 \mathrm{mM}$ EDTA in $\mathrm{Ca}^{2+}$ and $\mathrm{Mg}^{2+}$-free phosphate-buffered saline (PBS). For experiments, cells were resuspended in PBS, $\mathrm{pH}$ 7.4.

\section{Large Unilamellar Vesicles}

Lipids in a chloroform solution were dried in a round-bottom flask under vacuum. Lipid mixtures were prepared by codissolving in chloroform the appropriate proportions (expressed as $\mathrm{mol} / \mathrm{mol}$ ratio). Multilamellar liposomes (MLVs) were prepared by dispersing the dry lipid film in $150 \mathrm{mM} \mathrm{NaCl}$, Tris $20 \mathrm{mM}$, pH 7.0 solutions.

Large unilamellar vesicles (LUVs) were prepared from MLV suspension by extrusion through polycarbonate membranes of $100 \mathrm{~nm}$ diameter using a Liposofast equipment (Vancouver, Canada) (12).

\section{Merocyanine Fluorescence Spectrum}

Stock solutions of MC540 were prepared in ethanol to facilitate solubilization and stored at $-20^{\circ} \mathrm{C}$. An appropriate aliquot of $10^{-6} \mathrm{M}$ of MC540 in water was further used. MC540 spectra in the presence of LUVs or cells were obtained under different conditions in a SLM 4800 spectrofluorometer (SLM Instruments Inc. Urbana, IL). A ratio of $1: 200$ dye : lipid or $1: 200$ dye : cells $\left(10^{6} \mathrm{cell} / \mathrm{mL}\right)$ was prepared for these experiments, and after 30-s incubation periods, the fluorescence emission spectra at $540 \mathrm{~nm}$ wavelength excitation were recorded (13). 


\section{Steady-State Fluorescence Anisotropy}

Fluorescence anisotropy was determined using diphenylhexatriene (DPH) as a membrane probe. DPH in tetrahydrofurane was incorporated into LUVs by adding the appropriate aliquots in order to reach a probe : lipid molar ratio $1: 500$ and incubated at room temperature in darkness for $60 \mathrm{~min}$. All measurements were made at $25^{\circ} \mathrm{C}$. Fluorescence anisotropy was determined in a format T-SLM 4800 spectrofluorometer (SLM Instruments Inc. Urbana, IL) equipped with excitation and emission polarizers. Steady-state anisotropy was calculated using the equation

$$
r_{s}=\frac{I v v-I v h}{I v v+2 I v h}
$$

where $I_{\mathrm{vv}}$ and $I_{\mathrm{vh}}$ denote the intensities obtained with vertical and horizontal orientations of excitation polarizers. Excitation and emission wavelengths for DPH were 361 and $442 \mathrm{~nm}$, respectively (14).

\section{RESULTS AND DISCUSSION}

Merocyanine 540 is a naturally fluorescent lipophilic probe that has been used to investigate membrane surface properties of different cells and phospholipid vesicles. It has been reported that MC540 can distinguish between subtle differences in plasma membranes of very similar cells, for instance in the case of leukemic and nonleukemic limphocytes $(15,16)$.

The dye can be easily incorporated into bilayers of phospholipid model membranes and be partitioned into the lipid phase when it is added externally to an aqueous liposome suspension (13). MC540 exhibits an equilibrium between fluorescent monomers and nonfluorescent dimmers, which depends on the dispersing medium. In phospholipid bilayers, the monomer chromophore is thought to be located near the membrane-water interface. It resides slightly above the domain of the glycerol backbone of neutral and charged phospholipids, and it is here that the dye is very sensitive to structural alterations in the lipids. The dye binds preferentially to membrane crystalline phase, where its fluorescence intensity is greatly enhanced. Upon the membrane phase transition to the gel state, the fluorescence substantially decreases. In fact, it can be used to detect the phase transition of bilayers and to sense the packing degree of lipid membranes. MC540 binds preferentially to bilayers in which the lipids are disordered or loosely packed (17).

Figure 1 shows a representative spectrum of MC540 in aqueous solution as well as in the presence of cells before and after their incubation with $0.1 \mathrm{mM}$ vanadate. As it can be seen in this figure, the spectrum in aqueous solution has a low fluorescence with a wavelength maximum of about $575 \mathrm{~nm}$. In the presence of cells, the emission wavelength maximum 


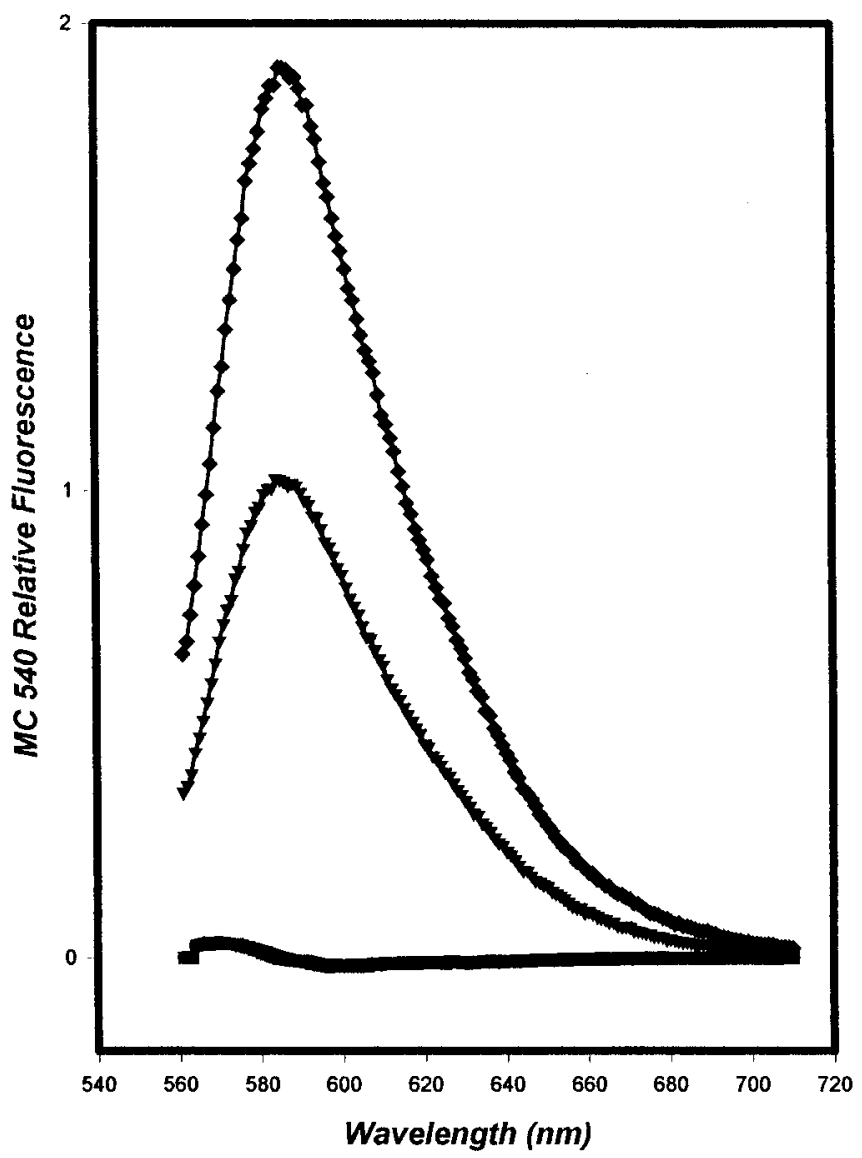

Fig. 1. Representative spectra of MC540 in buffer solution (control, $\mathbf{\square}$ ) and in the presence of UMR 106 cells before $(\boldsymbol{\nabla})$ and after $(\checkmark)$ adding $0.1 \mathrm{mM}$ vanadate. Cells were incubated with vanadate for $5 \mathrm{~min}$ and MC540 was added. The spectra were recorded after $30 \mathrm{~s}$ of the addition of the probes.

is shifted to about $587 \mathrm{~nm}$, which corresponds to a fluorescent monomer of MC540. Cells preincubated with $0.1 \mathrm{mM}$ vanadate for $5 \mathrm{~min}$ before the addition of MC540 exhibited a much greater fluorescence. Because the dye is impermeant, it is restricted to the plasma membrane when applied externally to cells. The results in Fig. 1 thus suggest an increased interaction of this probe with the external surface of vanadate-treated osteoblastic cells.

It has been previously reported that vanadate is a potent membrane permeable inhibitor of the aminotraslocase (18). The translocation mechanism is specific for aminophospholipids (phosphatidylethanolamine and phosphatidylserine) that are concentrated in the inner monolayer by an ATP-dependent process $(19,20)$. On the contrary, the choline phospholipids (sphingomyelin and phosphatidylcholine) are predominantly 
located in the outer monolayer (21). Increased binding of the dye to the vanadate-exposed cells could be a consequence for the loss of membrane asymmetry. The inner leaflet of the plasma membrane is originally more fluid than the outer one; consequently, loss of lipid asymmetry results in the externalization of lipids originally concentrated in the inner lipid leaflet. The outer lipid leaflet becomes less ordered; thus, it could enhance its affinity for MC540.

Alternatively, other mechanisms of interaction between vanadate and the membrane could be involved. In order to further investigate the direct effect of vanadate on the lipid membrane, a series of experiments were performed using POPC (LUV). This phospholipid was chosen to prepare LUVs because it is the predominant phospholpid species in the outer monolayer of the plasma membrane. The fluorescence spectrum of MC540 in the presence of POPC liposomes, which are in the liquidcrystalline state at $25^{\circ} \mathrm{C}$, shows a peak at $587 \mathrm{~nm}$ and it increases when LUVs are previously incubated with vanadate (data not shown). Figure 2 shows that the fluorescence intensity of MC540 at the emission maximum increases as a function of vanadate concentration in the media. This effect shows saturation at a concentration higher than $0.1 \mathrm{mM}$ vanadate, as seen in this figure. This effect could indicate a great disorder in lipid membranes exposed to vanadate and, consequently, an increase in the MC540 fluorescence. We could assume that the vanadate binding to lipid vesicles induces a separation of the phospholipid head groups and loose packing of the fatty acid region near the interface.

In order to gain a further insight into the vanadium-membrane interaction, the effects of a different vanadium compound were investigated. MC540 spectrum of POPC-LUV was obtained in the presence of vanadyl(IV) cation. In contrast to vanadate, vanadyl decreases the fluorescence of the MC540 monomer (Fig. 3). This observation suggests stabilization of the order in the lipid membranes of vesicles induced by vanadyl(IV). It is conceivable that $\mathrm{VO}^{2+}$ could build up bridges, similar to those proposed for divalent cation like $\mathrm{Ca}^{2+}$. The presence of negative charges, as in phosphate groups at the lipid interface, promotes the chelation of $\mathrm{Ca}^{2+}$ ions, inducing structural rearrangements in membrane stability, permeability, and surface potential $(22,23)$. In the same way, the structure of membrane could be stabilized by the interaction between $\mathrm{VO}^{2+}$ and the phosphate groups of the phospholipids.

With the aim of studying if a vanadium-membrane interaction affects the hydrocarbon core of the lipid bilayer, the effect of the vanadium compounds on the acyl chain of the vesicles was measured by using a DPH probe. The fluidity or microviscosity of membrane is often estimated from the steady-state fluorescence anisotropy of DPH incorporated into the hydrophobic bilayer (14). This parameter reveals the average angular displacement of the fluorophore that occurs between absorption and subsequent emission, depending on the rate and extent 


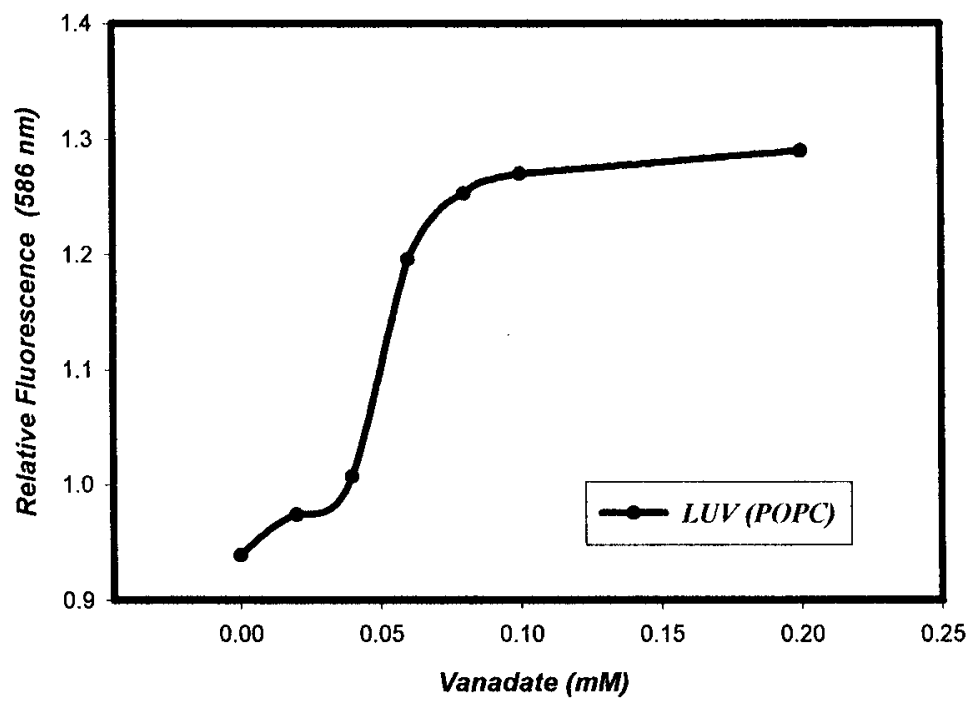

Fig. 2. MC540 fluorescence intensity at $586 \mathrm{~nm}$ incorporated into (POPC) LUV as a function of vanadate concentration. The final lipid concentration was $0.1 \mathrm{mM}$ and the dye : lipid ratio was $1: 200$ ( $\mathrm{mol}: \mathrm{mol})$. The spectrum was registered after $30 \mathrm{~s}$ of probe addition.

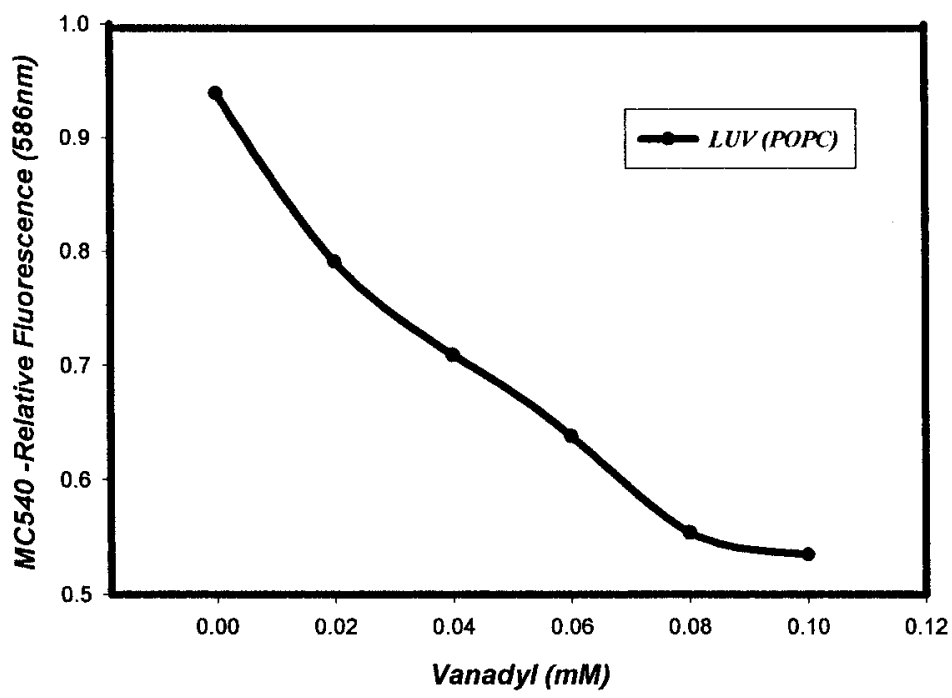

Fig. 3. MC540 fluorescence intensity at $586 \mathrm{~nm}$ incorporated into (POPC) LUV as a function of vanadyl concentration. The final lipid concentration was $0.1 \mathrm{mM}$ and the dye : lipid ratio was $1: 200$ (mol : mol). The spectrum was registered after $30 \mathrm{~s}$ of probe addition. 


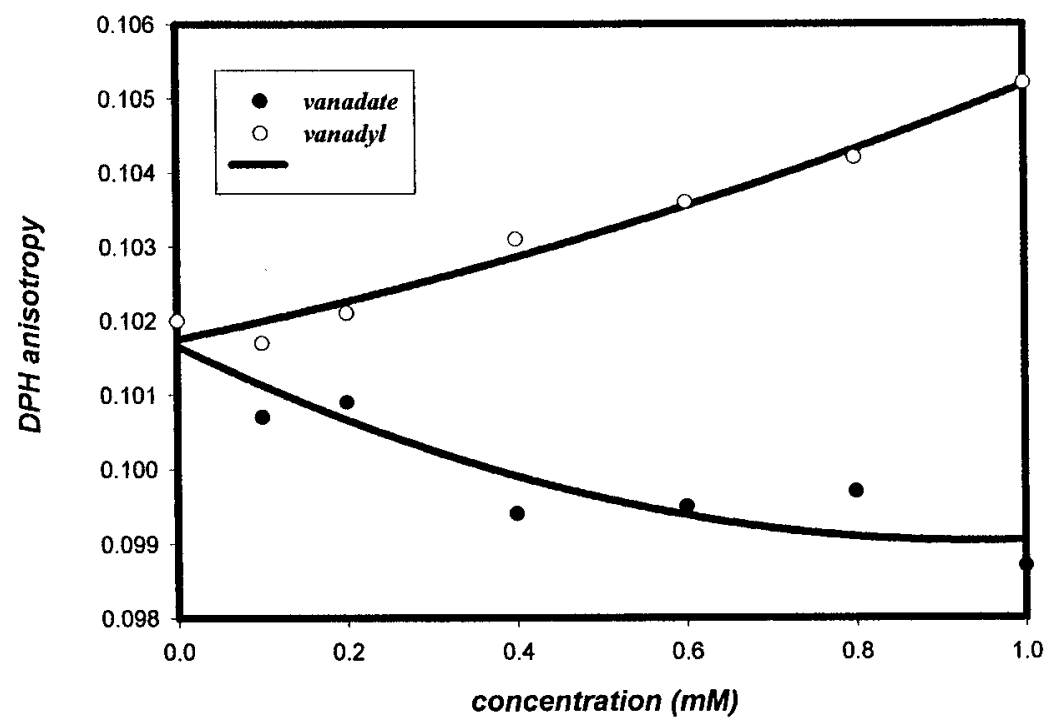

Fig. 4. Steady-state anisotropy of DPH incorporated into (POPC) LUV as a function of different concentrations of vanadate $(\mathbf{O})$ and vanadyl $(\bigcirc)$. The concentration of DPH was $10^{-6} \mathrm{M}$ in lipid membranes. The anisotropy was measured at $25^{\circ} \mathrm{C}$.

of rotational diffusion during the lifetime of the excited state (24). The addition of vanadate to POPC-LUV produces a slight but significant $(p<0.02)$ decrease in the fluorescence anisotropy from $0.102 \pm 0.001$ to $0.098 \pm 0.001$ in the absence or presence of $1 \mathrm{~m} M$ vanadate, respectively (Fig. 4). On the other hand, when vanadyl(IV) was incubated with POPC-LUV, the DPH anisotropy was increased in a dose-response manner from $0.102 \pm 0.001$ to $0.106 \pm 0.001(p<0.02)$. In contrast to the results obtained with MC540, $0.1 \mathrm{mM}$ vanadate did not induce important changes at the hydrocarbon core of lipid membranes. These results would indicate that vanadium compounds slightly perturb the hydrophobic probes and their action remains at the membrane surface.

The role of membrane composition, in particular the presence of phosphatidylserine on the membrane interactions with vanadium compound, is shown in Fig. 5. MC540 spectra were obtained in the presence of LUV composed of POPC: PS $(3: 1)$ molar ratio. The presence of negatively charged phospholipids decreases the vanadate-membrane interaction in comparison with the effect observed when POPC-LUV was employed. Moreover, the presence of PS in the vesicle promotes vanadyl interaction with the membranes, demonstrating the role of electrostatic interaction with the polar head groups of lipid membranes.

Vanadium is a transition metal that shows a flexible coordination sphere. Vanadate has available $d$-orbitals through which it could have a coordination number of $5,6,7$, or 8 . The major vanadate species under physiological conditions $\left(\mathrm{H}_{2} \mathrm{VO}_{4}^{-}\right)$has only one negative charge. 


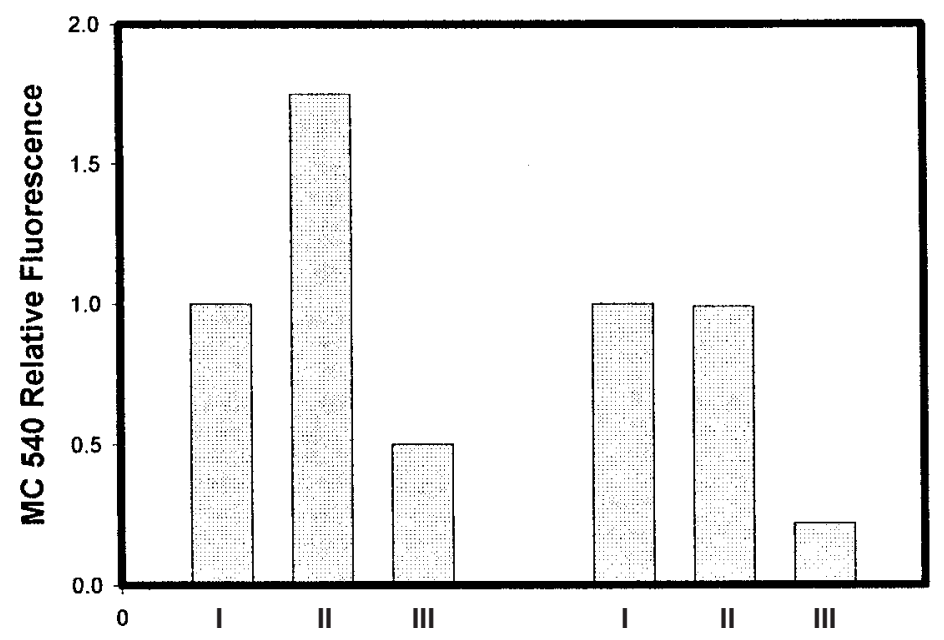

Fig. 5. MC540 relative fluorescence at emission maximum $(586 \mathrm{~nm})$ in the presence of (A) LUV(PC) or (B) LUV PS/PC (3:1, mol : mol). I, II, or III correspond to control, $0.1 \mathrm{mM}$ vanadate(V), and $0.1 \mathrm{mM}$ vanadyl(IV), respectively.

Metavanadate, obtained by dissolving $\mathrm{NaVO}_{3}$ in $\mathrm{H}_{2} \mathrm{O}$, is a mixture of monovanadates and oligovanadates, the composition of which depends on the total vanadium concentration, $\mathrm{pH}$ and ionic strength, and temperature of the medium. At vanadate concentrations below $0.1 \mathrm{mM}$, the vanadate monomer is the only species observed by ${ }^{51} \mathrm{~V}-\mathrm{NMR}$ (nuclear magnetic resonance) in aqueous solutions (25). All oligomeric species are anionic forms of vanadate at $\mathrm{pH} \mathrm{7.0,} \mathrm{and} \mathrm{they} \mathrm{could} \mathrm{interact} \mathrm{with} \mathrm{cationic}$ ligands like choline groups of phosphatidyl choline (PC). Monovanadates, divanadates, linear vanadates, tetravanadates, and decavanadates may be protonated at acidic $\mathrm{pH}$. However, under our experimental conditions, the oligomer formation is not probable. Thus, we could assume that the major species of vanadium $(\mathrm{V})$ interacting with the membrane is negatively charged with a four-coordination geometry. On the other hand, vanadyl(IV) could interact with different ligands in aqueous solution. Because of its high affinity for ligands like oxygen, nitrogen, and sulfur, the complexation of vanadyl(IV) enhances its solubility. Thus, interacting with the polar head group of phospholipid, it could induce a higher organization of the membrane. In the presence of PS, vanadyl induced a greater effect in comparison to PC alone. These results could suggest a specific interaction of vanadyl(IV) with the serine groups present in the membrane.

In this study, we have used relatively high concentrations of vanadium to investigate its acute effect on lipid membranes. It could be argued that this observation is unrelated to its function as an essential trace element in vivo. However, it is known that bone is a particular organ where vanadium is concentrated, the total-body storage of vanadium is about $100 \mu \mathrm{g}$ and the average daily intake is less than $50 \mu \mathrm{g} / \mathrm{d}$. 
The amount required for the biological effects of vanadium are between 50 and $100 \mathrm{mg} / \mathrm{kg}$ in animals and 100-125 mg in man. In osteoblastlike cells, we have previously shown that vanadium exerts several effects in a time- and dose-dependent manner. For instance, vanadium regulates growth and differentiation after $24 \mathrm{~h}$ incubation with doses of $2.5-100 \mu \mathrm{M}$ $(3,4,26)$. Vanadium also induces morphological transformation, oxidative stress, and protein tyrosine phosphorylation at a high concentration (0.1-5 $\mathrm{mM}$ ) when cells are treated for short periods (minutes to hours) $(27,28)$. This evidence thus suggests that the biological effects of vanadium are pharmacological and it has been suggested that vanadium be considered a drug rather than a supplement (29).

In conclusion, we have demonstrated a direct effect of vanadium compounds upon lipid membranes. The interaction of vanadium with phospholipids of the plasma membrane might have important consequences for the functional properties of the cells. The changes in the structural arrangement of the lipid bilayer of the membrane can, consequently, influence the functional properties of membrane-bound and peripheral proteins, which play an important role in morphogenesis and normal tissue remodeling through their interactions with the cytoskeleton.

\section{ACKNOWLEDGMENTS}

This work was partially supported by grants from Facultad de Ciencias Exactas, UNLP, CICPBA, and Agencia Nacional de Promoción Cientifica y Tecnologica (Pict 00375). LSB and AMC are members of the Carrera del Investigador, CICPBA, and GV is a fellow from CICPBA (Argentina).

\section{REFERENCES}

1. F. H. Nielsen and E. C. Uthus, The essentiality and metabolism of vanadium, in Vanadium in Biological Systems. N. D. Chasteen, ed., Kluwer Academic, Dordrecht, pp. 51-62 (1990).

2. S. B. Etcheverry and A. M. Cortizo, Bioactivity of vanadium compounds on cells in culture, in Vanadium in the Environment Part 1: Chemistry and Biochemistry, Wiley, New York, Vol. 15, pp. 359-393 (1998).

3. A. M. Cortizo and S. B. Etcheverry, Vanadium derivatives act as growth factormimetic compounds upon differentiation and proliferation of osteoblast-like UMR106 cells, Mol. Cell. Biochem. 145, 97-102 (1995).

4. S. B. Etcheverry, D. C. Crans, A. D. Keramidas, and A. M. Cortizo, Insulin-mimetic action of vanadium compounds on osteoblastic like cells in culture, Arch. Biochem. Biophys. 338, 7-14 (1997).

5. A. Stern, X. Yin, S. S. Tsang, A. Davison, and T. Moon, Vanadium as a modulator of cellular regulatory cascades and oncogene expresion, Biochem. Cell. Biol. 71, 103-112 (1993).

6. P. Lonnroth, J. W. Erkson, B. I. Poner, and U. Smith, Peroxovanadate but not vanadate exert insulin-like effect in human adipocytes, Diabetologia 36, 113-116 (1993).

7. R. W. Zhang, D. J. Simmons, R. S. Crowther, S. Mohan, and D. J. Baylink, Contribution of marrow stroma cells to the regulation of osteoblast proliferations in rats: evidence for the involvement of insulin-like growth factors, Bone Miner. 13, 201-215 (1991). 
8. B. R. Nechay, L. B. Nanninga, and P. S. Nechay, Vanadyl (IV) and Vanadate (V) binding to selected endogenous phospholipid carboxyl and amino ligands: calculation of cellular vanadium species distribution, Arch. Biochem. Biophys 51, 128-138 (1986).

9. D. C. Crans, R. L. Bunch, and L. A. Theisen, Interaction of trace levels of vanadium (IV) and vanadium (V) in biological systems, J. Am. Chem. Soc. 111, 7597-7607 (1989).

10. D. C. Crans, Interaction of trace levels of vanadium(IV) and vanadium (IV) in biological systems, Commun. Inorg. Chem. 16, 1-33 (1994).

11. E. J. Baran, Vanadyl (IV) complexes of nucleotides, in Metal Ions in Biological System, H. Sigel and A. Sigel (eds.), Dekker, New York, pp. 129-146 (1995).

12. R. Mac Donald, R. I. Mac Donald, B. M. Menco, K. Takeshita, N. Subbarao, and $\mathrm{L}$. $\mathrm{Hu}$, Small-volume extrusion apparatus for preparation of large unilamellar vesicles, Biochim. Biophys. Acta 858, 161-168 (1986).

13. A. S. Verkman, Mechanism and kinetics of MC 540 binding to phospholipid membranes, Biochemistry 26, 4050-4056 (1987).

14. M. Schinitzky and Y. Barenholz, Fluidity parameters of lipid regions determined by fluorescence polarization, Biochim. Biophys. Acta 519, 367-394 (1978).

15. J. E. Valinsky, T. G. Easton, and E. Reich, Morocyanine 540 as a fluorescence probe of membrane selective staining of leukemic and immature haemopoietic cells, Cells 13, 487-499 (1978).

16. G. Dodin and J. Dupont, Thermodynamics and kinetics of the interactions of MC540 with hydrophobic structures. Binding of MC540 to soy beam phosphatidylcholine oligolamellar liposomes and to mitochondria, J. Phys. Chem. 91, 6322-6326 (1987).

17. W. Stillwell, S. R. Wassall, A. C. Dumaual, W. D. Ehringer, C. W. Browning, and L. J. Jenski, Use of Merocyanine 540 (MC540) in quantifying lipid domains and packing in phospholipid vesicles and tumor cells, Biochim. Biophys. Acta 1146, 136-144 (1993).

18. L. C. Cantley, M. D. Resh, and G. Guidoti, Vanadate inhibits the red cells Na-K ATPase from the cytoplasmic side, Nature 272, 552-554 (1978).

19. A. Zachowski, E. Favre, S. Cribier, P. Herve, and P. Devaux, Outside-inside translocation of aminophospholipid in the human erythrocyte membrane is mediated by a specific enzyme, Biochemistry 25, 2585-2590 (1986).

20. D. L. Daleke and W. Huestis, Erythrocyte morphology reflects the transbilayer distribution of incorporated phospholipids, J. Cell Biol. 158, 1375-1385 (1989).

21. J. A. F. Op. Den Kamp, Lipid asymmetry in membranes, Annu. Rev. Biochem. 48, 47-71 (1979).

22. E. A. Disalvo and L. S. Bakas, Influence of the surface charge distribution and water layers on the permeability properties of lipid bilayers, in The Electrical Double Layer in Biology, M. Blank, ed., Plenum, New York, pp. 63-76 (1985).

23. L. S. Bakas and E. A. Disalvo, Asymmetric effect of the $\mathrm{Ca}^{+2}$ adsorption on the sonicated vesicles bilayers, Biochim. Biophys. Acta 979, 352-360 (1989).

24. J. Lackowicz, Principles of Fluorescence Spectroscopy, Plenum New York, pp. 357-359 (1984).

25. D. C. Crans, E. M. Willging, and S. R. Butler, Vanadate tetramer as the inhibiting species in enzymes reactions in vitro and in vivo. J. Am. Chem. Soc. 112, 427-432 (1990).

26. D. A. Barrio, M. D. Braziunas, S. B. Etcheverry, and A. M. Cortizo, Maltol complexes of vanadium (IV) and (V) regulate the alkaline phosphatase activity and the osteoblast-like cell growth, J. Trace Elements Med. Biol. 11, 110-115 (1997).

27. V. C. Sálice, A. M. Cortizo, C. L. Gómez Dumm, and S. B. Etcheverry, Tyrosine phosphorylation and morphological transformation induced by four vanadium compounds on MC3T3E1 cells, Mol. Cell. Biochem. 198, 119-128 (1999).

28. A. M. Cortizo, L. Bruzzone, S. Molinuevo, and S. B. Etcheverry, A possible role of oxidative stress in the vanadium-induced cytotoxicity in the MC3T3E1 osteoblast and UMR106 osteosarcoma cell lines, Toxicology, 147, 88-99 (2000).

29. S. Verna, M. C. Can, and J. H. McNeill, Nutritional factors that favorably influence the glucose/insulin system: vanadium, J. Am. Coll. Nutr. 17, 11-18 (1998). 